\title{
Non-Circulatory Force on a Finite Thickness Body Encountering a Gust
}

\author{
P. Gehlert* and H. Babinsky ${ }^{\dagger}+$ \\ University of Cambridge, Cambridge, United Kingdom, CB2 IPZ
}

The origin of the non-circulatory force arising during a cylinder sharp-edged gust encounter is investigated experimentally at a Reynolds number of 6000. Planar particle image velocimetry (PIV) and force balance measurements are employed simultaneously to assess the response of a translating cylinder encountering nominal gust ratios of $0.5,1$ and 1.5. Although the vortex sheet distribution, which represents the boundary layer vorticity, agrees with that calculated following Küssner's potential flow approach for a sharp-edged transverse gust, it was found that the sheet can also be determined from the vorticity distribution in the shear layers forming the gust edges. Furthermore, it is found that the non-circulatory force calculated using Küssner's model is an overestimate because the implicit assumption of rigid gust shear layers leads to an unphysical additional momentum change as a body of volume enters the gust regime. A 'volume correction' is therefore proposed. In addition, it is found that the gust shear layer width and deformation have a noticeable effect on the unsteady non-circulatory force development, which reduces the applicability of Küssner's method for large gust ratios and objects with considerable volume.

\section{Selected Nomenclature}

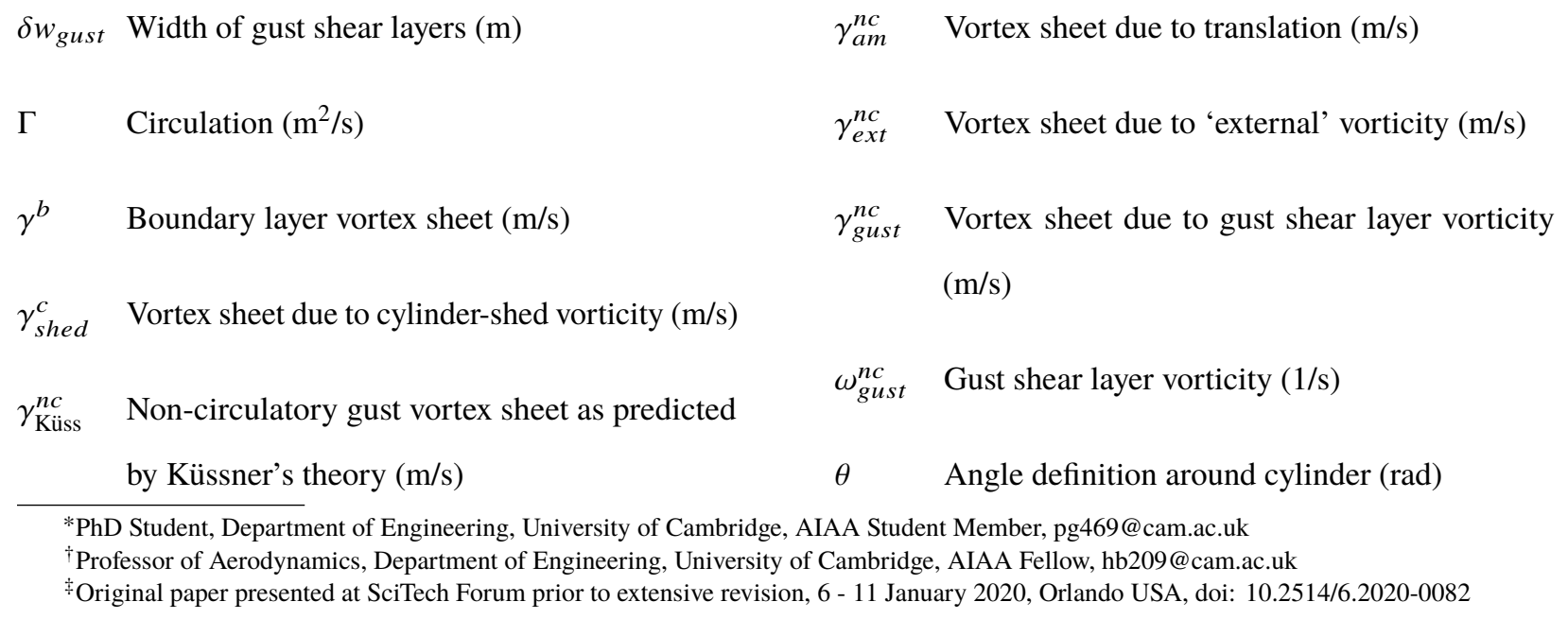

${ }^{*} \mathrm{PhD}$ Student, Department of Engineering, University of Cambridge, AIAA Student Member, pg469@cam.ac.uk

†Professor of Aerodynamics, Department of Engineering, University of Cambridge, AIAA Fellow, hb209@cam.ac.uk

† Original paper presented at SciTech Forum prior to extensive revision, 6 - 11 January 2020, Orlando USA, doi: 10.2514/6.2020-0082 


\begin{tabular}{|c|c|c|c|}
\hline$\widehat{C}_{l \text { Küssne }}^{n c}$ & Uncorrected non-circulatory Küssner lift coef- & $V$ & Transverse gust velocity $(\mathrm{m} / \mathrm{s})$ \\
\hline & ficient & $V_{c y l}$ & Volume per unit length of cylinder inside gust \\
\hline$a$ & Cylinder radius (m) & & $\left(\mathrm{m}^{2}\right)$ \\
\hline$c$ & Plate chord (m) & $C_{l}^{a d v}$ & Lift coefficient due to advection of gust shear \\
\hline$D$ & Cylinder diameter $(\mathrm{m})$ & & layer vorticity \\
\hline$G R$ & Gust Ratio & $C_{l_{\text {Küssner }}^{n c, s m e}}$ & eeared Smeared non-circulatory Küssner lift co- \\
\hline$I_{\text {gust }}^{\text {nc }}$ & Flow impulse from non-circulatory gust vortex & & efficient \\
\hline & sheet (Ns) & $C_{l} l_{\text {Küssner }}^{n c}$ & Corrected non-circulatory Küssner lift coeffi- \\
\hline$s$ & Cylinder position (m) & & cient accounting for the body volume effect \\
\hline$U$ & Instantaneous translation velocity $(\mathrm{m} / \mathrm{s})$ & $C_{l g u s t}^{n c}$ & Experimental non-circulatory gust lift coeffi- \\
\hline$U_{\infty}$ & Steady-state streamwise velocity $(\mathrm{m} / \mathrm{s})$ & & cient \\
\hline$U_{\gamma^{b}}$ & $\begin{array}{l}\text { Velocity induced by cylinder boundary layer } \\
(\mathrm{m} / \mathrm{s})\end{array}$ & $C_{l}^{n c} V_{c y l}$ & $\begin{array}{l}\text { Lift coefficient due to momentum change inside } \\
\text { cylinder }\end{array}$ \\
\hline
\end{tabular}

\section{Introduction}

7 RANSVERSE gusts are a ubiquitous feature in the atmospheric boundary layer, affecting the flight envelope of insects, birds and micro aerial vehicles (MAV) [1-3]. The low flight speeds of MAVs are a particular problem because they lead to high ratios between the transverse gust velocity and the flight speed. This parameter is commonly referred to as the gust ratio, GR. Large scale flow separation resulting from strong wing gust interactions can generate highly unsteady forces, imposing limitations on when MAVs can be deployed. As a result, research effort [4-6] has focused on understanding the unsteady flow field in order to develop control methods [7] to counteract the gust force. Furthermore, the need to mitigate gust forces extends beyond MAV's and equally applies to helicopters [8] and water turbines [9], where control methods can improve the safety of passengers and the longevity of equipment.

Simple closed-loop reactionary control unfortunately has an inherent time delay between the gust force and the mitigation effort, as control mechanisms are only employed once the gust force is already acting on the object. To improve the control outcome, a preemptive strategy is therefore ideally used. This requires models that can quickly predict the force, given some basic information about the gust. Low order models are ideal candidates for this because of their low computational cost enabling real-time predictions. They often rely on breaking down the force response into various components, each of which is described by a basic model. 
Insects, birds and MAVs often feature wings that can be approximated as infinitely thin flat plates and therefore much of the recent research has focused on this simple geometry. One approach to identify the different force contributions during an unsteady gust encounter is to make use of an idea from potential flow theory, where a flat plate immersed in a flow can be represented by a sheet of variable vorticity distributed along its surface [10]. The vortex sheet strength is equal to the slip velocity between the flow and the plate surface. Recently, authors [11-15] have successfully used this concept of vortex sheets to link force components to individual unsteady phenomena. This is possible because:

1) In a real viscous flow, the vorticity in the boundary layer is equivalent to the vortex sheet strength and can therefore be measured. Thus, although this concept arises from potential flow theory, it applies to a real flow, albeit with an implicit assumption that boundary layers are infinitely thin.

2) The vortex sheet surrounding the plate can be viewed as the linear superposition of a number of individual contributions, each linked to a different physical 'effect', as seen in figure 1 . In each case, the actual vortex sheet can be found either by considering the associated slip velocity or by enforcing the no-penetration condition due to a hypothetical flow field only containing this 'effect'. Any time-variation in the distribution of a vortex sheet component can subsequently be linked to a force [16].
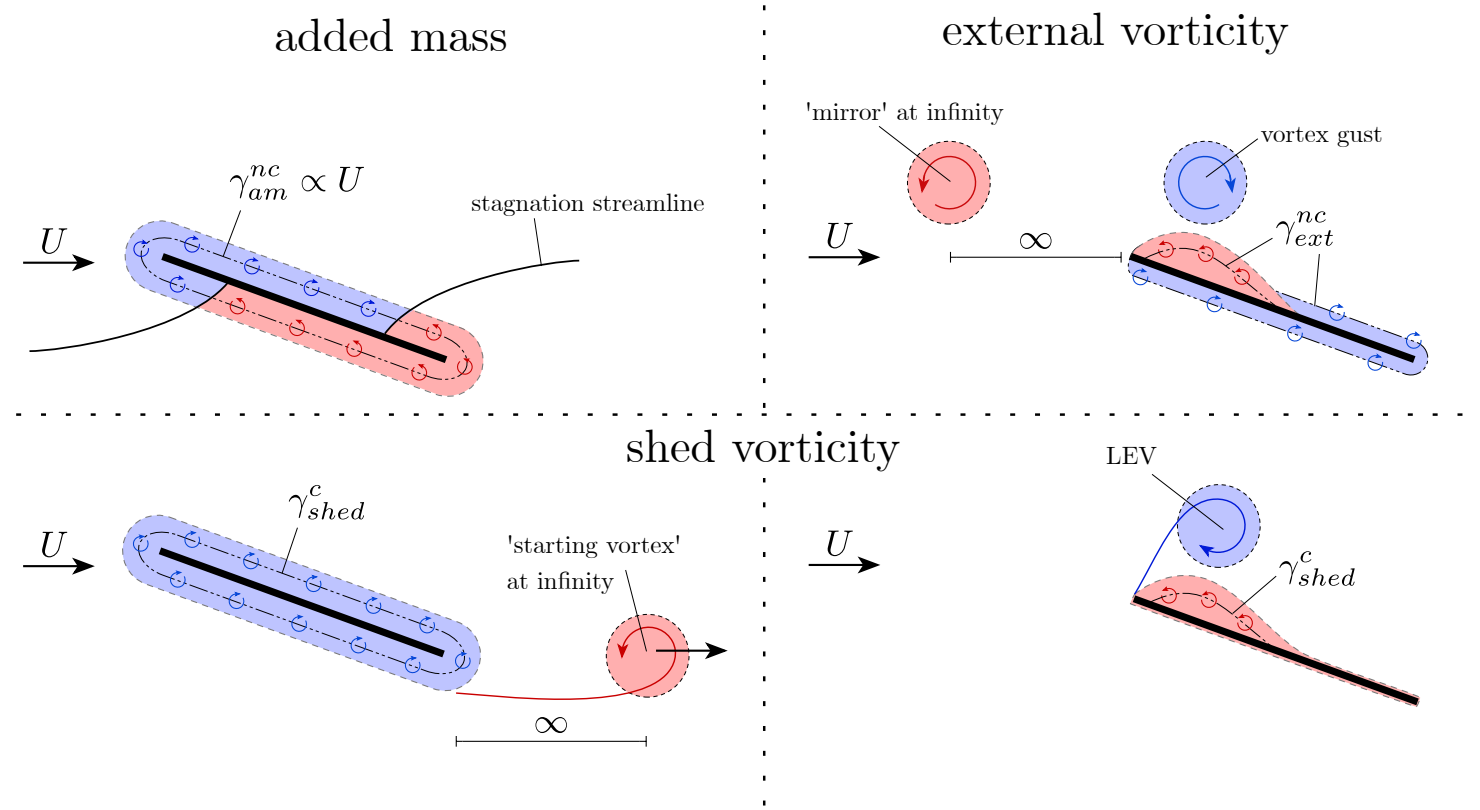

Fig. 1 Circulatory vortex sheet due to shed vorticity and non-circular contributions due to translation and external vorticity.

The superposition of all the individual flow contributions results in the true flow that is observed. For example, imagine a flat plate at an angle of incidence translating in quiescent fluid. One flow field component arises from the translation of the plate and can be computed from potential flow. The slip velocity between the inviscid flow field and the plate 
surface determines a vortex sheet, $\gamma_{a m}^{n c}$, as shown in the top left corner of figure 1 . The net circulation of the vortex sheet is zero and it is therefore categorised as a non-circulatory vortex sheet. Initially, while the plate is at rest, $\gamma_{a m}^{n c}$ is zero everywhere. As it accelerates to a velocity $U$, the vortex sheet grows to some distribution. Therefore, whilst the vortex sheet evolves, a force is created which is referred to as the added mass force. The existence of this vortex sheet and its link to the added mass force has been verified experimentally for rotating and accelerating flat plates by Corkery \& Babinsky [13].

In viscous flows, vorticity is likely to be shed at the edges of the plate, as schematically illustrated in the lower half of figure 1. This shedding leads to the development of an additional vortex sheet, $\gamma_{\text {shed }}^{c}$ which can be understood as the 'mirror image' of the shed vorticity. Any variation of its distribution, regardless of whether it changes in strength or redistributes at the same overall magnitude, results in a further force. Since the circulation of this vortex sheet is equal and opposite to that of the shed vorticity and is therefore not necessarily zero, the associated force is referred to as circulatory.

Similarly, if vorticity that was not originally shed by the plate is present in the flow then this 'external vorticity' generates a 'mirror' vortex sheet contribution, $\gamma_{e x t}^{n c}$, on the plate surface. In general, the global flow field is assumed to be irrotational and therefore, the external vorticity will have a counterpart of opposite sign elsewhere in the flow [17]. This will also contribute to the plate vortex sheet and causes the total circulation of $\gamma_{e x t}^{n c}$ to vanish; therefore, $\gamma_{\text {ext }}^{n c}$ is considered to be non-circulatory.

To apply these ideas to a gust, Küssner [18, 19] and von Kármán \& Sears [20] investigated a flat plate at zero angle of incidence entering a sharp edged gust of semi infinite extent. Von Kármán \& Sears decomposed the force into a component associated with shed circulation and a second linked to a non-circulatory vortex sheet residing on the plate surface, $\gamma_{\text {Küss }}^{n c}$. They show that this non-circulatory vortex sheet develops while the plate enters the gust, as schematically illustrated in figure 2. Once the plate is fully immersed in the gust, the vortex sheet distribution is exactly the same as if the plate were in a transverse freestream equal to the gust velocity, as shown in figure 3 Therefore, von Kármán \& Sears called the force created by the rate of change of $\gamma_{\text {Küss }}^{n c}$ an apparent or added mass force. 


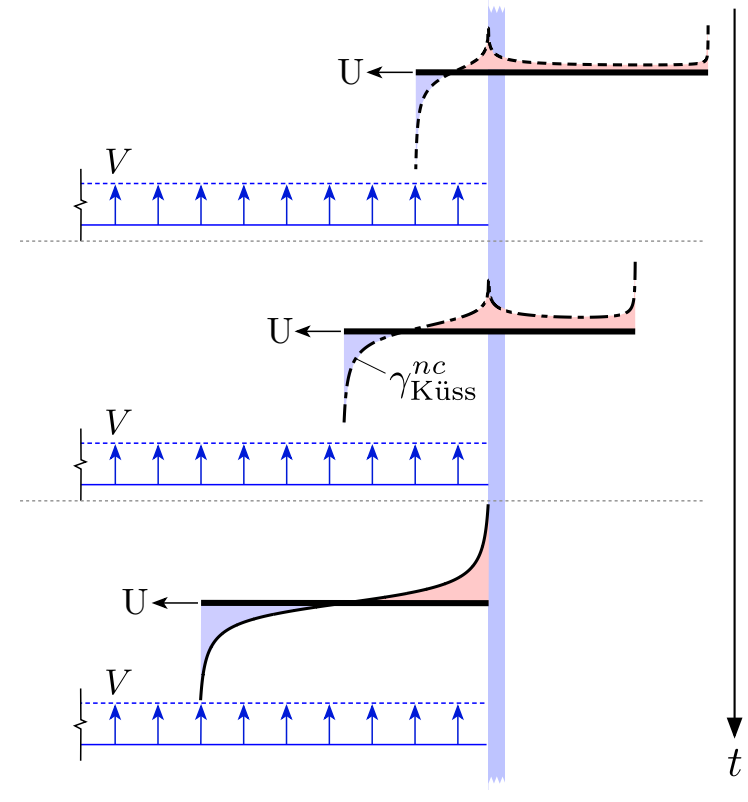

Fig. 2 Schematic illustration of a thin flat plate entering a gust and its $\gamma_{\text {Küss }}^{n c}$ distribution.

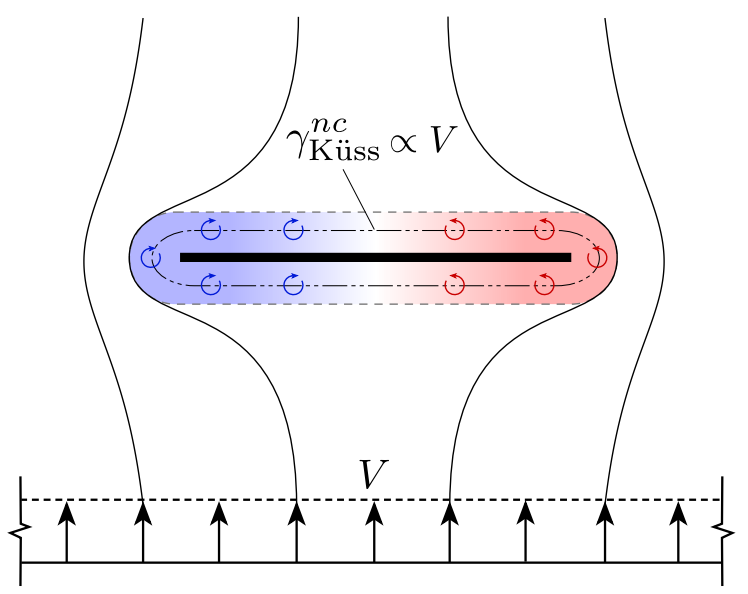

Fig. 3 Flow field around a plate immersed in a transverse gust is identical to a transverse freestream.

However, the developmental process of $\gamma_{\text {Küss }}^{n c}$ is different to $\gamma_{a m}^{n c}$ and for thin flat plates, Corkery \& Babinsky [15] showed that $\gamma_{\text {Küss }}^{n c}$ can also be understood as the 'mirror' image of the gust shear layer vorticity, as schematically illustrated in figure 4 This effectively treats the gust edges as regions of external vorticity and thus the associated vortex sheet $\gamma_{g u s t}^{n c}$ can be seen as a special case of $\gamma_{\text {ext }}^{n c}$.

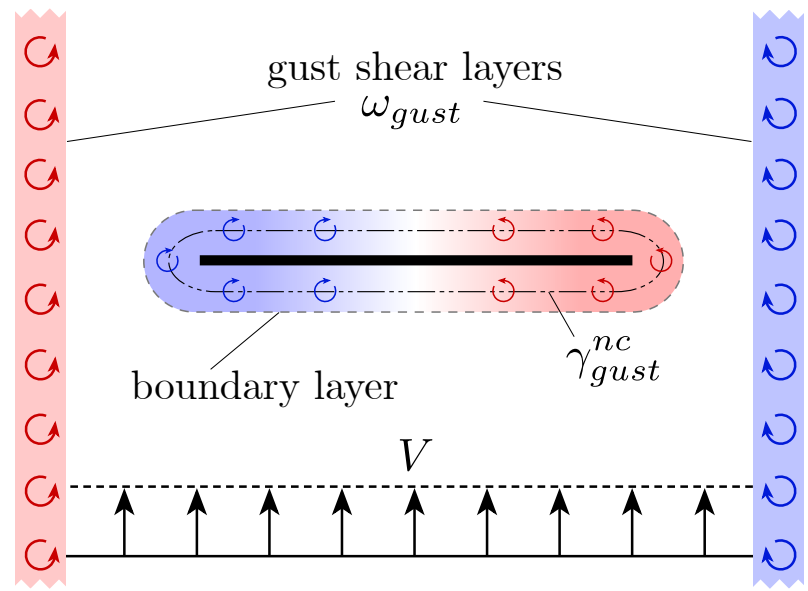

Fig. $4 \quad \gamma_{g u s t}^{n c}$ due to gust shear layer vorticity.

Even though the vortex sheet distributions and resulting force acting on the flat plate arising from $\gamma_{g u s t}^{n c}$ and $\gamma_{\text {Küss }}^{n c}$ are 
the same, the underlying origin is not. It is important to be aware of this in order to not double count the non-circulatory gust force and vortex sheet contribution. The total boundary layer vortex sheet, $\gamma^{b}$, is the sum of its individual contributions.

$$
\gamma^{b}=\gamma_{a m}^{n c}+\gamma_{\text {shed }}^{c}+\underbrace{\gamma_{\text {gust }}^{n c}}_{\gamma_{\text {gus }}} .
$$

As discussed $\gamma_{a m}^{n c}$ stems from plate translation and $\gamma_{s h e d}^{c}$ is due to any vorticity shed by the plate itself. The noncirculatory contribution due to the gust is obtained by further considering the gust shear layer vorticity. Since $\gamma_{g u s t}^{n c}$ and $\gamma_{\text {Küss }}^{n c}$ are identical, the effect from the gust, as proposed by von Kármán \& Sears, is already accounted for only by interrogating the complete flow field vorticity. Explicitly adding $\gamma_{\text {Küss }}^{n c}$ would result in its effect being counted twice.

The ability to successfully break down the unsteady force contributions into circulatory and non-circulatory components for a thin flat plate gust encounter is an important stepping stone in the development of low-order models. However, many realistic shapes, such as helicopter blades or water turbines, cannot be assumed to have negligible volume.

The aim of this paper is therefore to explore whether a non-circulatory vortex sheet, matching the potential flow distribution from a top-hat shaped transverse gust, can be recovered experimentally for bodies of volume and whether this is a consequence of the gust shear layer vorticity. If this is confirmed the respective force can be evaluated and compared to the theoretical equivalent calculated following Küssner's approach assuming a perfect gust. Comparing the experimental and theoretical non-circulatory gust force contributions, the effect of shear layer thickness and deflection can further be assessed.

To test these ideas on objects of volume, a circular cylinder with a thickness-to-chord ratio of 1 will be used. This is a simple shape, yet is far removed from an infinitely thin flat plate and therefore explores the extremes of the parameter space between an infinitely thin object and a body where the thickness is a non-negligible contribution to the geometry. In turn, the aerodynamic response of most objects is therefore expected to lie within these two extremes.

Furthermore, it should be noted that to simplify the analysis, a strictly two-dimensional approach is used throughout. Consequently, any presented force coefficient calculations assume a unit span and any reference to the volume of the cylinder is per unit length. Moreover, the flow field is idealised to be identical everywhere along the cylinder span. 


\section{Theoretical Sharp-Edged Gust Encounter}

\section{A. Küssner Gust Force for Bodies With Volume}

The Küssner model was derived for flat plates entering a sharp-edged gust. To simplify the problem, a number of inherent assumptions are made [20]. The wing is at zero angle of attack. Any perturbations due to the transverse gust are small, thus making linear thin airfoil theory applicable. Vorticity is shed from the wing only at the trailing edge and remains on the same horizontal plane. Furthermore, the delimiting gust edges are considered to be infinitely thin and rigid. Depending on the gust velocity and the shape of the body, these assumptions may be valid to more or less of an extent. However, a significant departure is to be expected for high gust ratios and bodies with thickness, as is the case for a cylinder.

We now begin to evaluate the Küssner model applied to a cylinder sharp-edged gust encounter. As explained in the following, the assumption of rigid non-deforming gust shear layers results in an erroneous additional force for bodies of volume.

The theoretical non-circulatory gust vortex sheet for a cylinder created by the gust shear layers can be obtained with a panel method approach. The cylinder surface is deconstructed counter-clockwise into a series of panels. A vortex as well as collocation point, where the no-through flow condition is enforced, are placed one quarter and three quarters along each panel. A transverse velocity $V$ is set at the collocation point for any panel that resides within the gust, whereas $V=0$ for all others. Thereafter, similar to point vortex models for example described by Spalart [21], a system of equations is simultaneously solved to arrive at the required vortex strength needed to enforce the no-penetration condition at all collocation points.

A schematic illustration of the gust encounter is provided in figure 5a, and the resulting vortex sheet distribution at various time steps during the gust entry is shown in figure $5 \mathrm{~b}$. It is observed that, similar to the flat plate case seen in figure 2, the vortex sheet distribution varies as the cylinder enters the gust. Once the cylinder is fully immersed in the gust, the vortex sheet distribution is, as expected, equivalent to that of a circular cylinder in cross-flow. Assessing the rate of change of the vortex sheet attributed to the gust shear layers gives the related non-circulatory Küssner force

$$
\widehat{C}_{l \text { Küssner }}^{n c}=\frac{d I_{\text {gust }}^{n c} / d t}{1 / 2 U^{2} D} .
$$

$U$ is the translation velocity and $D$ the cylinder diameter. $I_{g u s t}^{n c}$ represents the flow impulse, which for a vertical force, is defined as

$$
I_{g u s t}^{n c}=\int_{\hat{S}} \gamma_{g u s t}^{n c} x d l
$$




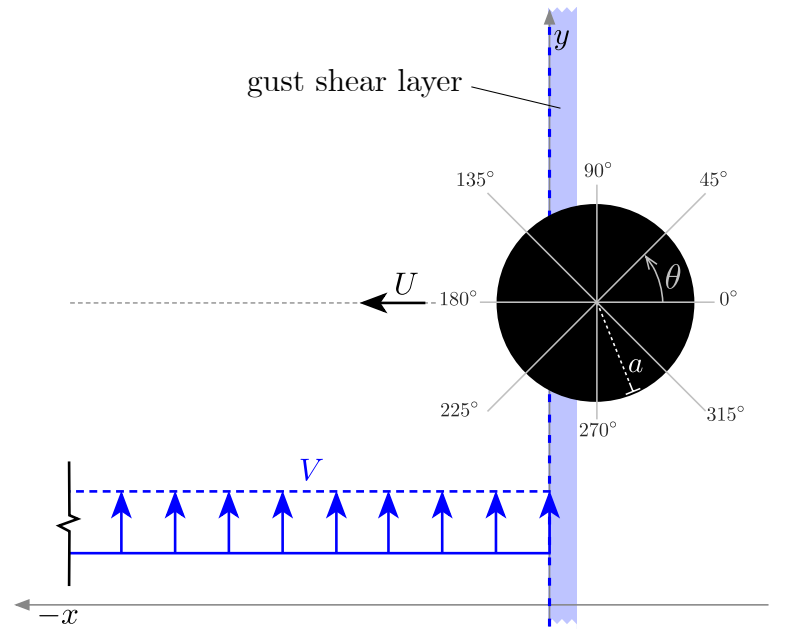

(a) Geometry and angle definition around cylinder surface.

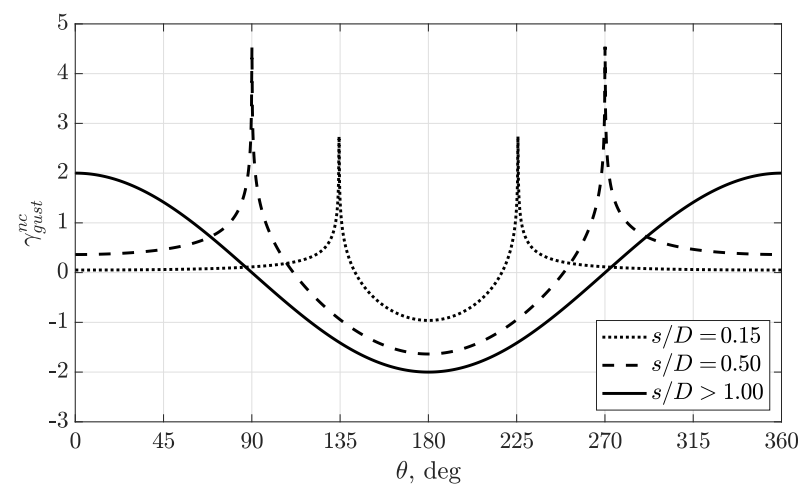

(b) Non-circulatory vortex sheet.

Fig. 5 Cylinder entering a rigid non-deforming gust.

where $x$ is the coordinate aligned with the horizontal axis and the integration is performed over the surface $\hat{S}$ of the cylinder. For the cylinder, the rate of change of the non-circulatory gust vortex sheet results in the uncorrected non-circulatory Küssner force $\widehat{C}_{l \text { Küssner }}^{n c}$ shown in figure 6 . For a top-hat gust encounter note that $\widehat{C}_{l_{\text {Küssner }}}^{n c}$ in figure 6 is only non-zero while the cylinder intersects the gust shear layers.

While the cylinder is outside, there is uniform transverse flow momentum inside the gust domain. However, as the cylinder encroaches on the gust, it begins to occupy some of its volume. By doing so, any transverse gust momentum in the region occupied by the cylinder is replaced by horizontal momentum related to the translation velocity of the cylinder, as schematically shown in figure 7 .

Looking closely at the flow field when the cylinder intersects the gust shear layer, one may notice that the unphysical rigid shear layer assumption leads to possible regions of non-zero fluid velocity within the cylinder, when the flow due to the gust and that induced by the related gust vortex sheet $\gamma_{\text {gust }}^{n c}$ are superimposed. This is because some gust shear layer vorticity is assumed to reside within the cylinder, which of course does not accurately represent reality. However, the regions of positive and negative flow within the cylinder cancel and lead to zero net flow within the cylinder, thus causing the above statement to remain valid.

Therefore, as each new section of the cylinder enters the gust, more of the transverse gust momentum is removed. The rate at which this occurs can in turn be linked to a force. In reality the momentum occupied by the cylinder is, of course, not 'lost' but rather re-distributed as the gust flow deflects around the cylinder. Unfortunately, the rigid shear layer assumption does not allow for this and hence, results in an additional force contribution. This can be corrected by removing the additional force due to the momentum change caused by the cylinder volume. This force coefficient due to 
this body volume effect is:

$$
C_{l \delta V_{c y l}}^{n c}=\frac{2 G R \frac{d V_{c y l}}{d t}}{U_{\infty} D}
$$

where $\frac{d V_{c y l}}{d t}$ is the rate at which the volume of the cylinder inside the gust changes and $G R$ is the gust ratio.

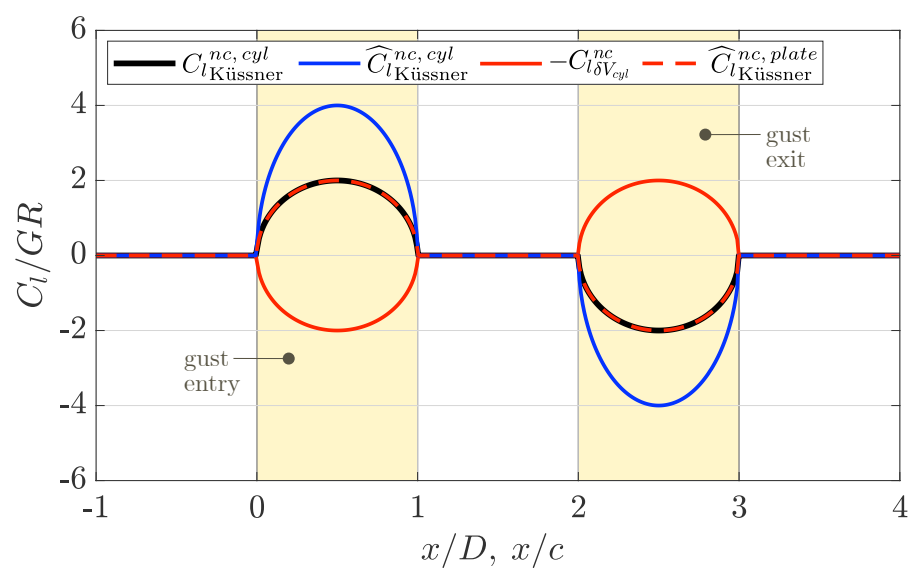

Fig. 6 Force coefficient contributions as the cylinder or plate intersects the rigid gust shear layers when entering and exiting a top-hat gust.

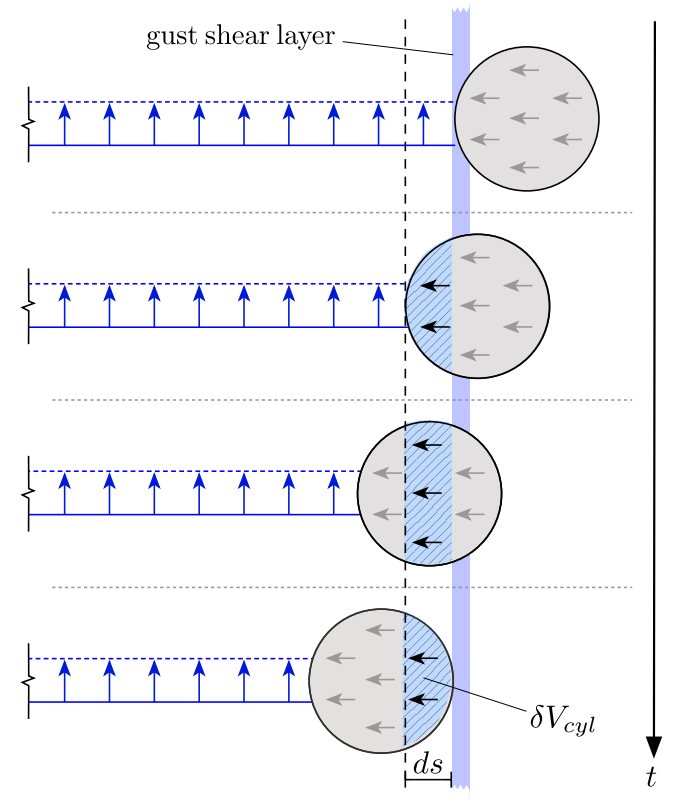

Fig. 7 Schematic illustration indicating the change in transverse momentum as the cylinder enters the gust.

The contribution due to the 'lost' transverse momentum inside the cylinder $C_{l_{\delta V_{c y l}}^{n c}}^{n c}$ turns out to be exactly half of $\widehat{C}_{l \text { Küssner }}^{n c}$, as seen in figure 6 Removing its contribution from $\widehat{C}_{l \text { Küssner }}^{n c}$,

$$
\begin{array}{r}
C_{l_{\text {Küssner }}^{n c}}^{n c}=\widehat{C}_{l_{\text {Küssner }}^{n c}-}-C_{l \delta V_{c y l}}^{n c} \\
\text { effect of lost gust momentum }
\end{array}
$$

results in the same non-circulatory Küssner force as calculated for a thin flat plate, as shown in figure $6 . C_{l_{\text {Küssner }}^{n c}}$ will be referred to as the corrected Küssner force for a circular cylinder, where the effect due to lost gust momentum as a result of body volume, has been accounted for.

\section{B. Accounting for Gust Idealisations}

The assumption of rigid and infinitely thin gust shear layers inherent in Küssner's approach may yield further sources of discrepancy with an actual gust encounter: 
- A realistic transverse gust is not perfectly sharp-edged. Instead, viscous effects thicken the gust shear layers and the gust velocity is reached over a short distance, $\delta w_{\text {gust }}$. This implies that the actual gust entry starts somewhat earlier and spreads over a longer distance, which results in a different (lower) force than predicted by Küssner's model.

- The gust shear layers deflect when the cylinder is in close proximity. This changes the gust velocity distribution and thus the force.

- Due to flow entrainment there may also exist a small yet a non-zero transverse velocity outside the gust. As the cylinder translates it may thus experience a small non-zero lift force before it reaches the upstream gust shear layer.

A schematic illustration of the these effects and the corresponding velocity distributions is shown in figure 8 .

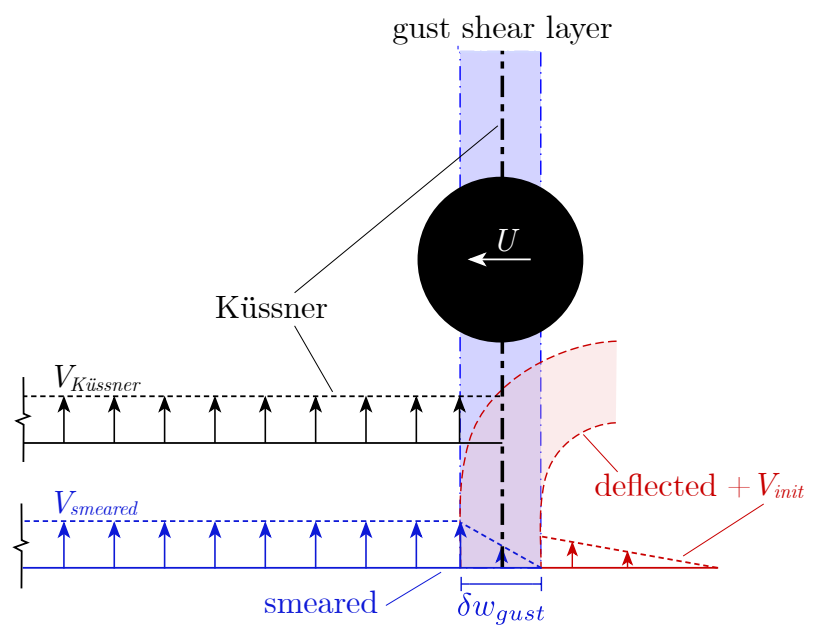

Fig. 8 Variations of the gust shear layer: The velocity profile for the Küssner and smeared gust are indicated as well as the non-zero velocity outside the gust.

\section{Effect of Gust Shear Layer Thickness}

The thickness of the shear layers can be accounted for theoretically by using the Duhamel integral. The gust velocity profile is split up into a number of discrete top-hat shaped gusts. Each of these discrete gusts contributes to the overall force coefficient acting on the cylinder according to

$$
K(s / D)=4 G R \sqrt{s / D-(s / D)^{2}}
$$

as derived by von Kármán \& Sears [20]. Superposing the effect from each element as the cylinder enters the gust recovers the force coefficient due to the smeared gust,

$$
C_{l_{\text {Küssner }}^{n c, \text { smeared }}}^{\text {nas }}=\int_{0}^{s / D} \frac{d K(\sigma)}{d \sigma} \phi(s / D-\sigma) d \sigma .
$$


Here $\phi(s / D-\sigma)$ ranges from 0 to 1 and corresponds to the gust velocity profile, $\sigma$ is a non-dimensional position inside the gust. It is assumed that to a first order the velocity rise occurs linearly over the shear layer width, from zero velocity outside the gust to the target velocity inside the gust. The resulting gust velocity profile and the non-circulatory gust force for a range of shear layer widths, $\delta w_{\text {gust }}$, is plotted in figure g⿵ It can be seen that with increasing gust shear layer width, the initial rise in $C_{l}$ is less rapid and has a lower maximum than the corrected non-circulatory Küssner force. It should be noted that the transverse velocity upstream of the first gust shear layer $V_{\text {init }}$ can equally be modelled using a Duhamel approach.

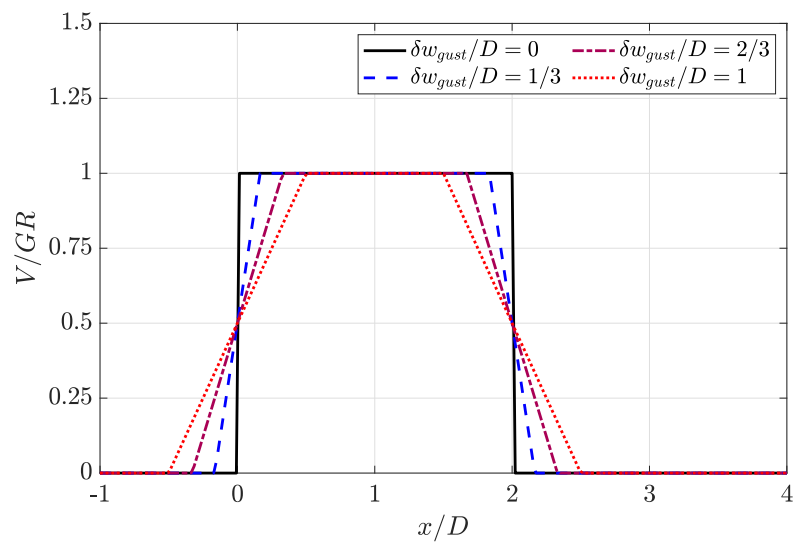

(a) Transverse gust velocity profiles for various gust shear layer widths.

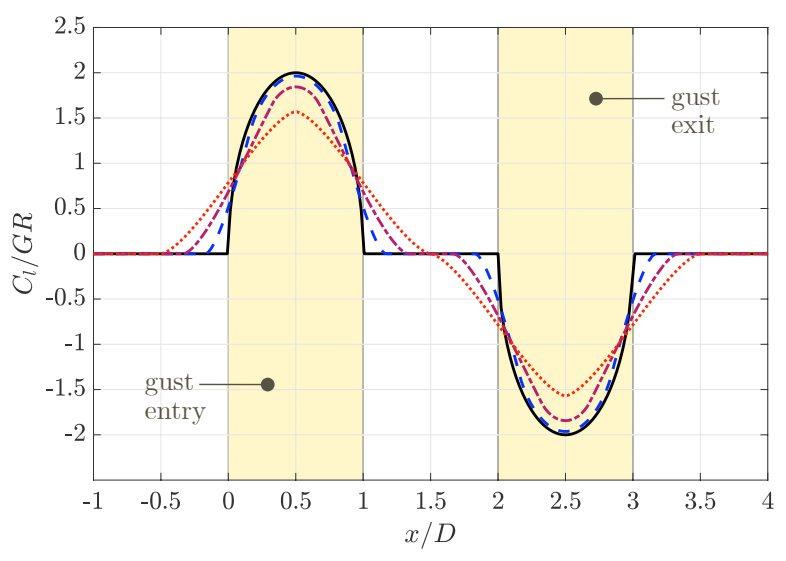

(b) Non-circulatory gust force. Gust entry and exit assuming infinitely thin gust shear layer are indicated.

Fig. 9 Effect of smeared gust shear layers.

\section{Effect of Gust Shear Layer Deflection}

In a real gust, the shear layers are not rigid but can deflect. This can happen for example due to the influence of a cylinder as is illustrated in figure 10 . The corresponding change in flow field momentum contributes a force coefficient that acts on the cylinder. Using the ideas outlined in [10, 22, 23], this associated force coefficient due to shear layer deflection can be calculated as follows:

$$
C_{l a d v}=\frac{\int_{A_{g u s t}^{n c}} \omega_{g u s t}^{n c} U_{\gamma^{b}} d A}{1 / 2 U^{2} D}
$$

where the integration is performed over the area associated with the gust shear layers, $A_{g u s t}^{n c} . \omega_{g u s t}^{n c}$ is the gust shear layer vorticity and $U_{\gamma^{b}}$ is the velocity induced by the cylinder boundary layer at each location of the flow field corresponding to the gust vorticity.

The total non-circulatory force acting on the cylinder due to the gust is therefore, the sum of the contribution due to the

\footnotetext{
${ }^{*}$ The rate of change of momentum inside the cylinder has also been accounted for as described in II.A
} 
rate of change of $\gamma_{g u s t}^{n c}$, equation 2 , and due to the advection of the gust shear layers,

$$
C_{l \text { gust }}^{n c}=\widehat{C}_{l \text { Küssner }}^{n c}+C_{l a d v} .
$$
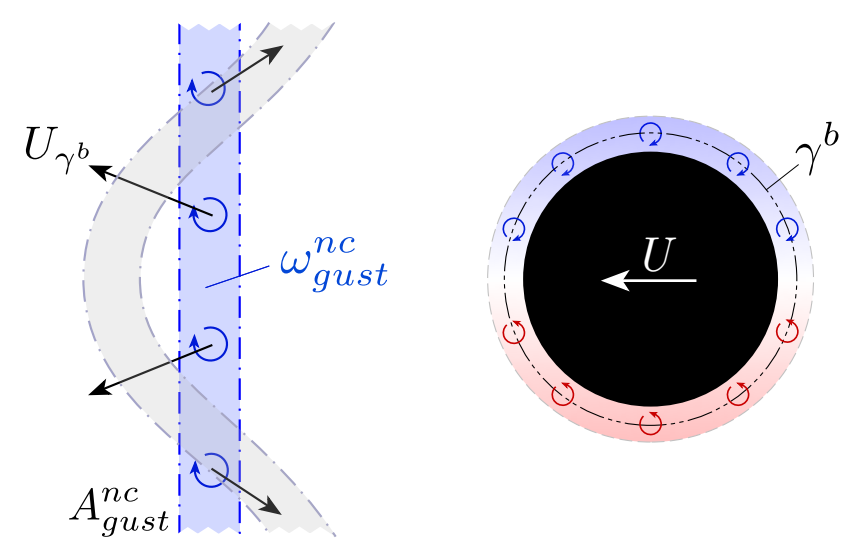

Fig. 10 Schematic illustration of the cylinder boundary layer inducing a velocity onto a gust shear layer. Subsequent deformation may result in a force.

To assess the significance of shear layer deflection, the theoretical non-circulatory gust force calculated assuming rigid shear layers can be compared to its experimental counterpart. To remove the effect of shear layer thickness, the experimental results can be compared to the smeared non-circulatory Küssner force.

\section{Experimental Cylinder Gust Encounter}

Having explored the origin of the boundary layer vorticity components as well as the relevant forces from a theoretical and potential flow perspective, an experimental cylinder gust encounter is investigated to test the validity of the proposed ideas.

\section{A. Experimental Set-Up}

The towing tank facility at the University of Cambridge is used to experimentally recreate the cylinder gust interaction. The $9 \mathrm{~m}$ long and $1 \mathrm{~m}$ wide tank is filled with water up to $0.8 \mathrm{~m}$ and the cylinder is vertically mounted to a force balance as shown in figure 11. The cylinder is $0.06 \mathrm{~m}$ in diameter and $0.48 \mathrm{~m}$ long. The clear acrylic is painted matte black everywhere apart from a small section at midspan to allow a laser sheet to pass through and illuminate the entire flow 
field. The cylinder connects to the force balance via an aluminium shaft attached at the top.

The servo motor driven carriage moves along the length of the tank. The gust rig, specifically designed for the facility, produces a region of transverse flow which the cylinder enters to create a sharp-edged gust encounter. A schematic illustration of the set-up is shown in figure 11 .

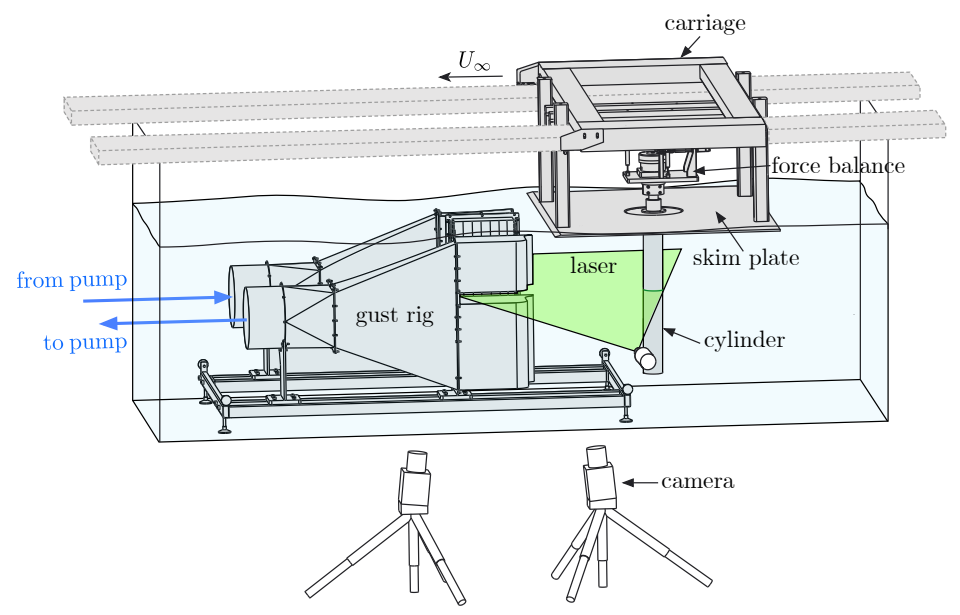

(a) Gust rig and cylinder arrangement.

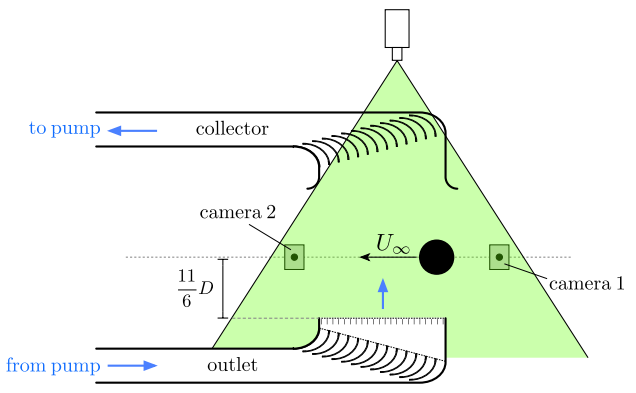

(b) Top view of PIV set-up.

Fig. 11 Experimental set-up.

Planar particle image velocimetry is conducted to assess the flow. Titanium dioxide particles are illuminated by a Nd:YLF $527 \mathrm{~nm}$ laser and two high-speed Phantom M310 cameras are positioned in a dual-camera arrangement to ensure optical access to all regions of the flow field. The sampling rate is $300 \mathrm{~Hz}$ for the individual test cases and 5 runs are conducted per kinematic case from which an ensemble average is obtained. The cross-correlation process, using the commercial LaVision Flowmaster 2D system, features an adaptive interrogation window with a size of $16 \times 16$ pixels during its final pass with an overlap of $50 \%$.

The gust rig consists of an outlet and a collector, which are connected to each other via a pump generating the flow. They create a flow perpendicular to the motion of the carriage. The top of the outlet is $0.03 \mathrm{~m}$ below the top of the cylinder. This distance is required to allow the skim plate, which is flush with the cylinder top, to pass over the gust rig. The cylinder centre is offset by $0.11 \mathrm{~m}$ from the gust outlet; equivalent to $\frac{11}{6} \mathrm{D}$, as illustrated in figure $11 \mathrm{~b}$ A gap in the collector allows for the laser sheet to pass through and illuminate the flow at the midspan of the cylinder as shown in figure $11 \mathrm{~b}$. Surface reflections of the laser sheet cause missing data in close proximity to the cylinder. As outlined in [14], the missing vorticity data is recovered by interpolating between the known cylinder surface velocity and the measured flow field. This preserves the magnitude of vorticity located directly around the cylinder but leads to an error in the surface-normal distribution of the vorticity. The problems due to reflections only extend approximately $0.02 D$ 
into the flow field. Therefore, the error in the distribution of wall normal vorticity is minor and does not adversely affect the results.

To ensure that peak-locking effects do not dominate the root mean square (RMS) velocity error estimation [24], the size and magnification of particles is adjusted so that the particle diameters are smeared over more than 2 pixels. According to Raffael et al. [25], this leads to a conservative velocity error estimate of $0.6 \%$ when the average particle displacement between consecutive frames is 3 - 4 pixels. The shift in the correlation peak, when an interrogation window is mapped back to its original position according to the calculated displacement vector, can also be used to estimate the measurement uncertainty [26]. The associated uncertainty is calculated by DaVis, and after being reduced according to $1 / \sqrt{N}$, where $N$ is the number of repeated runs [27], is $3 \%$. The total uncertainty of the velocity measurements is therefore, below $4 \%$.

The vorticity contours of the gust are shown in figure 12a The path taken by the cylinder centre as it traverses the gust is also indicated. The transverse velocity profile normalised by the average gust velocity, $v / \bar{V}$, is shown in figure $12 \mathrm{~b}$ at various stream-normal locations inside the gust. It is observed that the gust shear layers diffuse with distance from the gust outlet, leading to a less sharp gust profile. Note that the variation in gust velocity through the shear layer is not too different from the linear profile assumed earlier in section II.B.1. At the location where the cylinder crosses the gust, the shear layer width, $\delta w_{\text {gust }}$, is approximately $0.5 \mathrm{D}$. Furthermore, as alluded to in section II.B, a small yet non-zero velocity $V_{\text {init }}$ is recorded outside the gust. A more detailed description of the gust rig and its respective flow field can be found in [28] .

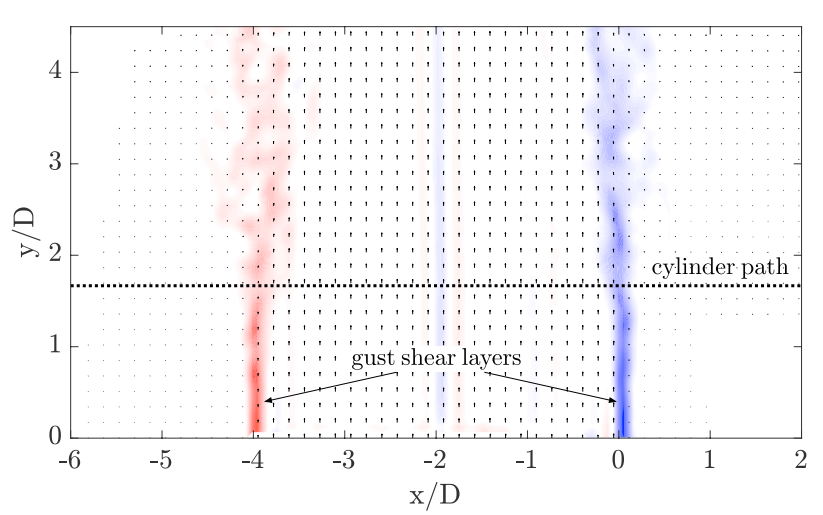

(a) Vorticity contours of the gust flow field.

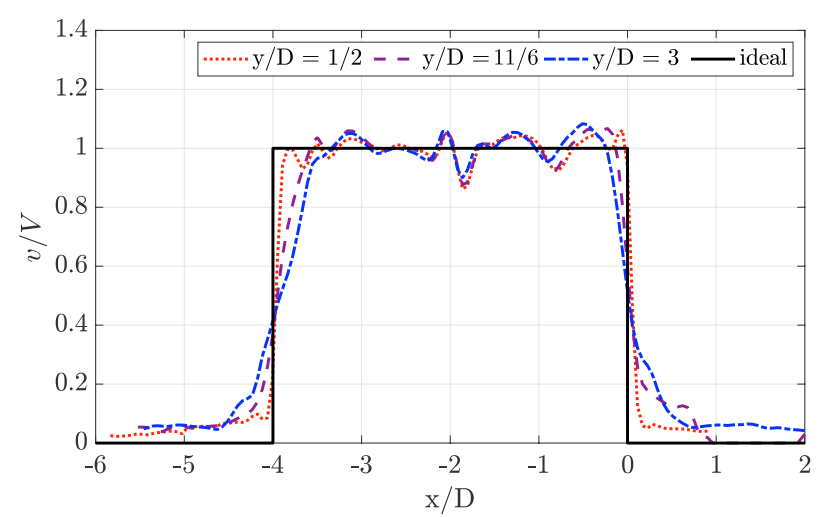

(b) Velocity profile of gust at various locations from the outlet.

Fig. 12 Gust flow field.

The forces acting on the cylinder are recorded with a Flow Dynamics two-component load cell. It allows force measurements between $\pm 50 \mathrm{~N}$ with a resolution of $0.01 \mathrm{~N}$ and is calibrated in-situ to obtain a representative force curve slope. The carriage position is obtained using an electro-optical sensor with a resolution of $1 \mathrm{~mm}$. The velocity is 
determined through numerical differentiation of the position data, whilst the acceleration is measured by an ADXL335 3-component micro-electromechanical accelerometer mounted to the carriage. All measurements are sampled at a rate of $3 \mathrm{kHz}$.

The force data are averaged over 5 runs and a zero phase shift averaging window is applied to reduce the effects of electrical noise and vibrations. For all cases an estimate of the standard random error relative to the peak mean force is below $2 \%$. The variation of the translation velocity during a run introduces an associated uncertainty of $1 \%$. This leads to a total uncertainty of approximately $3 \%$ associated with the final force coefficients.

Three different test cases are investigated, each consisting of a different cylinder kinematic and gust ratio combination. The Reynolds number, $R e$, based on the final cylinder surge velocity, is 6000 for all cases. The cylinder centre is initially located $1.4 D$ upstream of the gust outlet. From a stationary start, it proceeds to accelerate within $0.5 D$ to its final velocity of $0.129 \mathrm{~ms}^{-1}$. The test cases are summarised in table 1 . The gust experiments were performed at nominal gust ratios of $0.5,1$ and 1.5. The actual gust velocities, however, differed slightly from the target value, as indicated in table 1. Any cylinder forces presented are therefore normalised by the actual gust ratio.

Table 1 Summary of the test cases.

\begin{tabular}{rcccc}
\hline \hline Case & $\boldsymbol{G R}_{\text {true }}$ & $\boldsymbol{G R}_{\text {nominal }}$ & $\boldsymbol{U}_{\boldsymbol{\infty}}$ & $\boldsymbol{R} \boldsymbol{e}$ \\
\hline $\mathbf{1}$ & 0.42 & 0.5 & & \\
$\mathbf{2}$ & 0.94 & 1.0 & 0.129 & 6000 \\
$\mathbf{3}$ & 1.55 & 1.5 & & \\
\hline \hline
\end{tabular}

\section{B. Extracting $\gamma_{g u s t}^{n c}$}

To isolate $\gamma_{\text {gust }}^{n c}$, the approach outlined by Corkery \& Babinsky [15] is adapted to the current problem geometry.

The generic flow field around a cylinder immersed in a sharp-edged gust is shown in figure 13 . Vorticity resides in the gust shear layers and is also shed by the cylinder itself. As stated in equation 1 , the boundary layer vortex sheet surrounding the cylinder is made up of one component associated with the translation velocity, $\gamma_{a m}^{n c}$, a further arises

due to shed circulation, $\gamma_{\text {shed }}^{c}$, and a final contribution comes from the gust shear layer vorticity, $\gamma_{g u s t}^{n c}$. These can be re-arranged to isolate the contribution due to the gust shear layer vorticity:

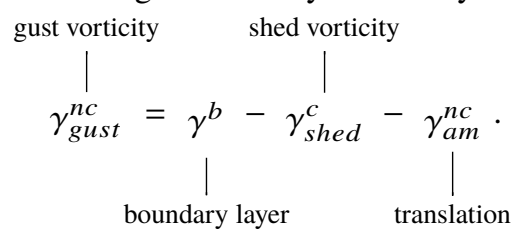




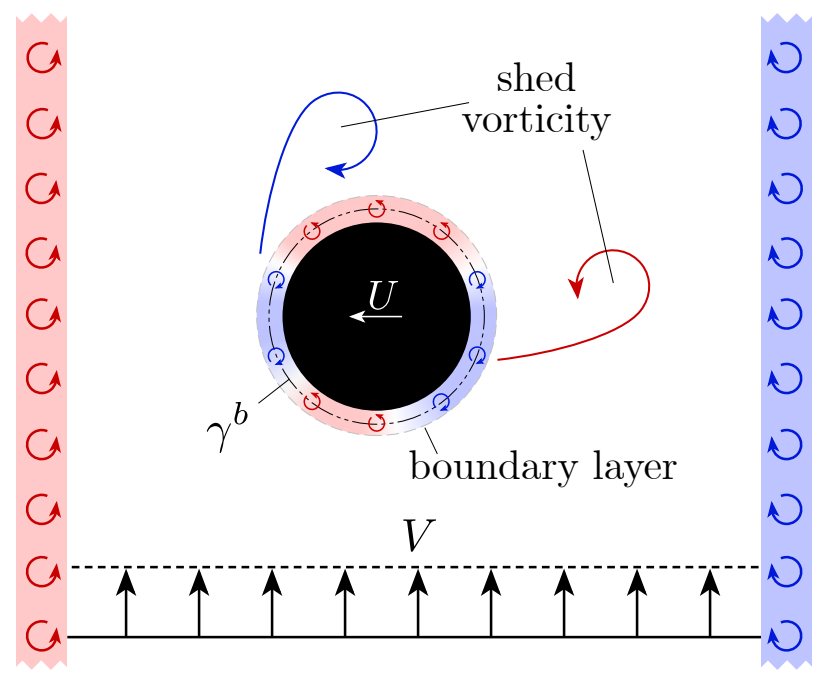

Fig. 13 Schematic illustration of a cylinder inside an idealised rigid top-hat shaped gust.

Once fully immersed in the gust, $\gamma_{\text {gust }}^{n c}$ can be calculated theoretically from knowledge of the transverse gust velocity distribution and is $2 V \cos \theta$. This is equal to the vortex sheet on a cylinder in transverse freestream.

To experimentally isolate $\gamma_{\text {gust }}^{n c}$, the boundary layer vorticity is recorded in order to obtain $\gamma^{b}$, and vorticity shed by the cylinder is identified to determine $\gamma_{\text {shed }}^{c}$. Furthermore, $\gamma_{a m}^{n c}$, which formed when the cylinder initially accelerated to its steady-state velocity is found from potential theory and equal to $-2 U \sin \theta$. The theoretical distribution of $\gamma_{a m}^{n c}$ has been recovered experimentally for a surging and rotating cylinder [14], verifying that it is, indeed, a contribution to the boundary layer vorticity and present in a real viscous flow.

To measure the boundary layer vorticity, $\gamma^{b}$, the cylinder circumference and surrounding flow field are split up into a number of wedges, as shown in figure 14 . The circulation of each segment, $\delta \Gamma_{n}=\oint u d l$, is found by integrating the velocity, $u$, aligned with the contour of each wedge. The velocity along the surface of the cylinder is set to the true physical surface velocity obtained by tracking the translation velocity. Dividing $\delta \Gamma_{n}$ by the segment length $\delta s_{n}$ yields the vortex sheet strength for each element ${ }^{\dagger}$

$$
\gamma_{n}=\frac{\delta \Gamma_{n}}{\delta s_{n}}
$$

$\gamma_{\text {shed }}^{c}$ is identified by manually masking the flow field to remove the gust shear layers. This process is rather straightforward when the cylinder is fully inside the gust, and an example of this is shown for Case 1 in figure 15 . However, it is more challenging when the cylinder is entering or leaving the gust. As the cylinder intersects the gust

\footnotetext{
Note that it is not necessary to fully resolve the boundary layer velocity profile in order to recover $\gamma^{b}$. Velocity vectors close to the surface will be almost tangential to the surface. Their contribution will therefore cancel. Effectively, it suffices to measure the velocity difference between a point just outside the boundary layer and the body surface velocity.
} 
shear layers, it can become difficult to distinguish between cylinder and gust shear layer vorticity and any results during this phase should therefore be treated with caution. Misattributed vorticity in close proximity to the cylinder can have a significant effect on the resulting vortex sheet distribution. In comparison, small portions of incorrectly assigned vorticity that are further away have less of an effect, as their is impact is small compared to that of the remaining far-field gust shear layer vorticity. Once the gust vorticity has been masked, $\gamma_{\text {shed }}^{c}$ is subsequently identified by finding the velocity imposed on the cylinder surface due the shed vorticity and the corresponding mirror counterparts as explained in [14].

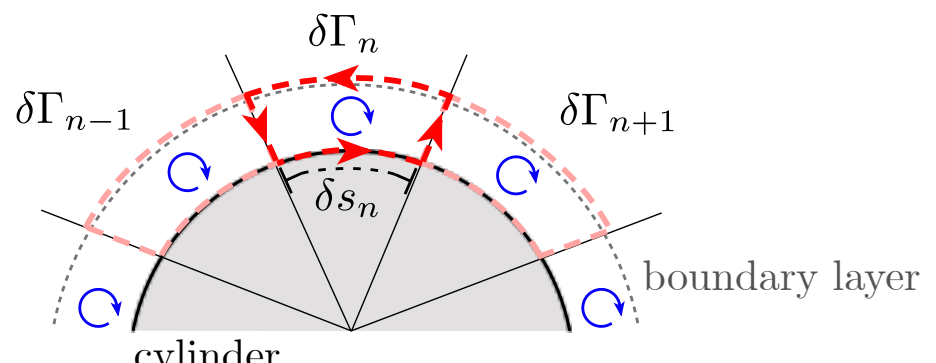

cylinder

Fig. 14 Discretization of the cylinder and surrounding flow field to obtain $\gamma^{b}$ from experimental data.

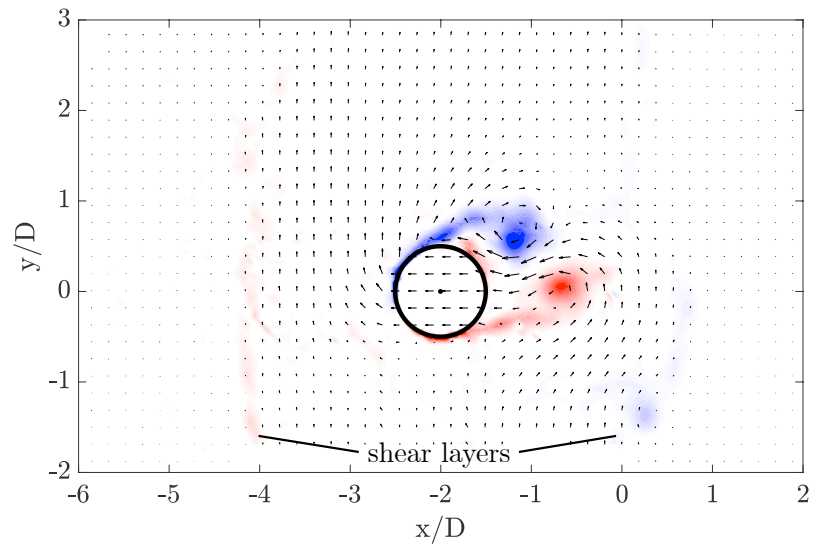

(a) Vorticity of complete flow field, $2 D$ inside gust.

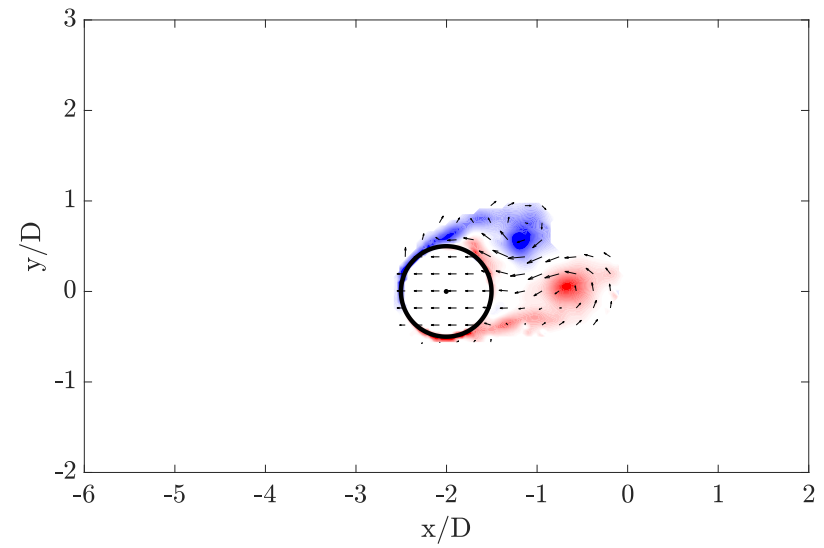

(b) Gust vorticity is masked, $2 D$ inside gust.

Fig. 15 Normalised vorticity field used to calculated $\gamma_{\text {shed }}^{c}$ for Case $1, G R=0.5$.

$\gamma_{\text {gust }}^{n c}$ does not vary when the cylinder is completely inside the gust, regardless of cylinder position. As a result, the measurement of $\gamma_{\text {gust }}^{n c}$ can be averaged over all time instances whilst the cylinder is immersed in the gust; reducing the noise in the data. Figures $16 \mathrm{a}$ through $16 \mathrm{c}$ show the results for the individual test cases. It is observed that $\gamma_{g u s t}^{n c}$ matches the theoretical distribution obtained from potential flow theory extremely well in all cases. Some small discrepancies can be seen between $270^{\circ}<\theta<320^{\circ}$ and around $\theta=135^{\circ}$. These locations correspond to the regions where flow separation occurs. Due to the nature of how the shed vorticity leaves the cylinder surface, some of the vorticity in close 
proximity to the cylinder is incorrectly associated with $\gamma^{b}$ instead of with $\gamma_{\text {shed }}^{c}$, and therefore causes local errors in the experimentally recovered $\gamma_{g u s t}^{n c}$ distribution. These errors can be as high as approximately $20 \%$ and from a quantitative perspective are therefore large. However, from a qualitative standpoint, they do not deter from the overall good match between the theoretical and experimental vortex sheet distribution, as these errors are only associated with small, limited regions along the cylinder surface.

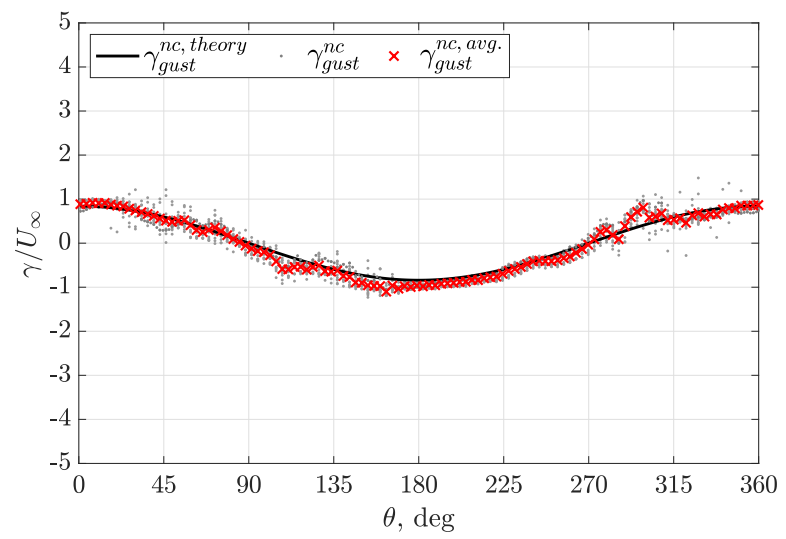

(a) Case 1, GR = 0.5.

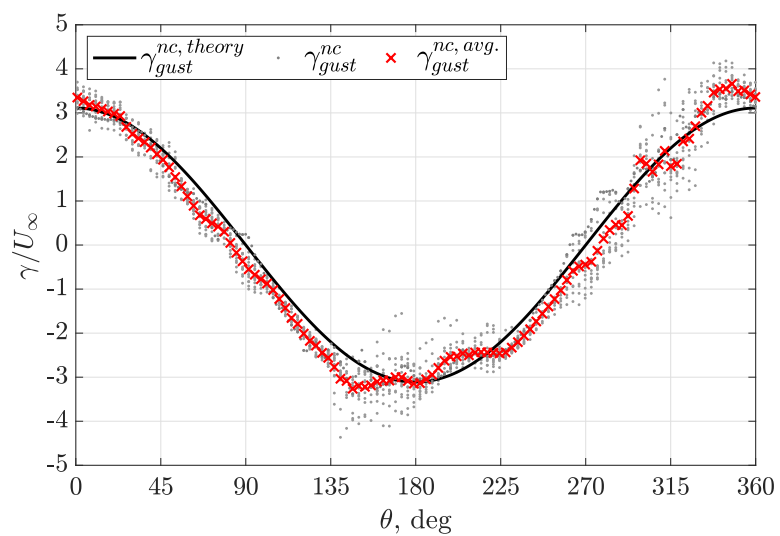

(c) Case 3, GR = 1.5.

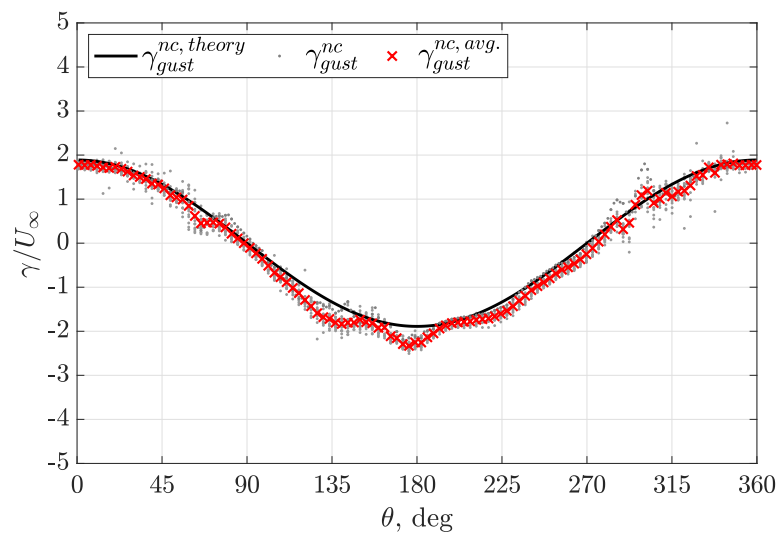

(b) Case 2, GR = 1 .

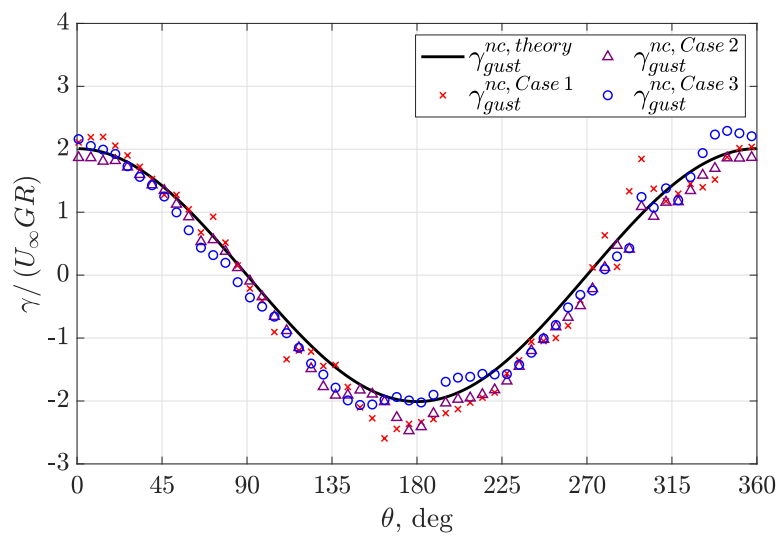

(d) $\gamma_{g u s t}^{n c}$ for all cases normalised by $G R$.

Fig. 16 Comparison between the theoretical and experimental distribution of $\gamma_{g u s t}^{n c}$.

Figures 16a through 16c confirm that $\gamma_{\text {gust }}^{n c}$ can be extracted experimentally by determining the bound vortex sheet and subtracting the vortex sheet contributions due to cylinder-shed vorticity and the translational velocity. Furthermore, the magnitude of $\gamma_{\text {gust }}^{n c}$ is directly proportional to the gust velocity. This is confirmed by figure $16 \mathrm{~d}$ which shows that all results from figures $16 \mathrm{a}-16 \mathrm{c}$ collapse and agree well with the theoretical prediction when they are non-dimensionalised by gust ratio. 
To determine whether $\gamma_{g u s t}^{n c}$ can be understood as a consequence of gust shear layers for a circular cylinder (which has volume), analogous to the findings of Corkery \& Babinsky for flat plates [15], the gust shear layer vorticity can be identified and the resulting vortex sheet distribution, following the same approach used for $\gamma_{\text {shed }}^{c}$, can be calculated.

The field of view measured by PIV, however, only covers a small portion of the gust shear layers. For the purpose of determining their impact on the cylinder vortex sheet, they are therefore augmented by point vortices at the same $x$-location extending for a further 60 cylinder diameters to model the uniform transverse gust. Figure 17a shows the additional point vortices together with the flow field after masking all vorticity shed from the cylinder. This remaining vorticity is used to compute $\gamma_{\text {gust }}^{n c}$ and the results are given in figure $17 \mathrm{~b}$ The blue symbols show the vortex sheet calculated from the measured gust shear layer vorticity only, while the red crosses include the effect of the additional point vortices. Using only the measured vorticity yields a distribution that is correct in shape but of the wrong magnitude. Incorporating the additional point vortices in the computation recovers the theoretical vortex sheet.

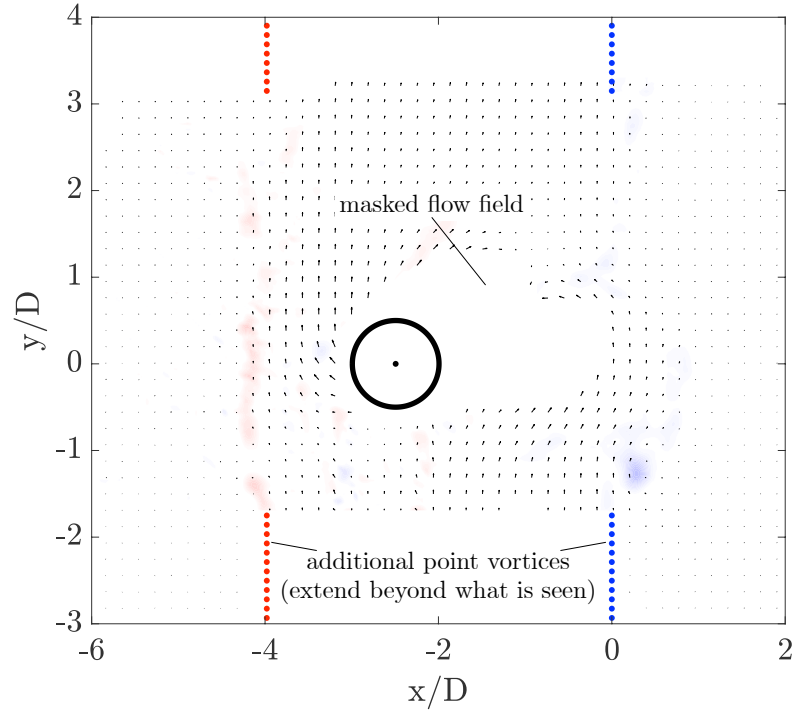

(a) Masked vorticity field used to calculate $\gamma_{g u s t}^{\text {nc }}$.

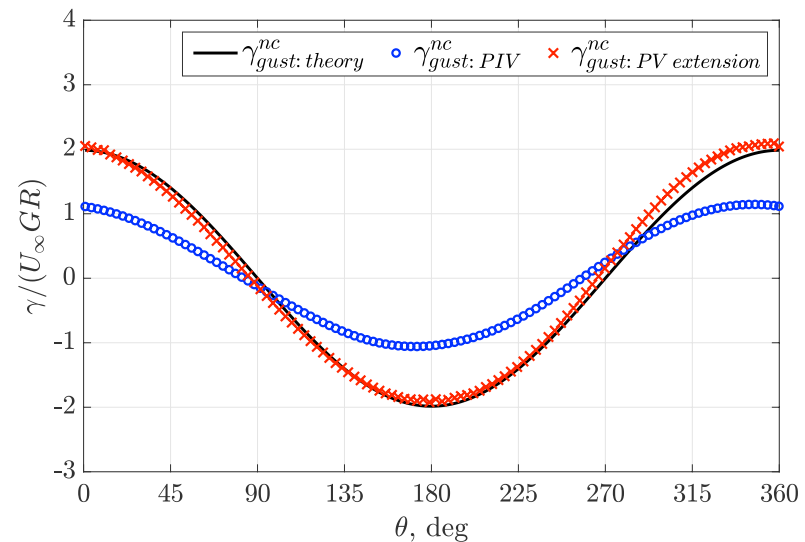

(b) $\gamma_{\text {gust }}^{\text {nc }}$ from vorticity field.

Fig. 17 Flow field and non-circulatory gust vortex sheet $\gamma_{g u s t}^{n c}$ as a result of extending the gust shear layers and masking any cylinder-shed vorticity. Case 1.

This clearly demonstrates that the non-circulatory gust vortex sheet recovered from a knowledge of the gust shear layer vorticity distribution is identical to the vortex sheet attributed to an added mass effect by von Kármán \& Sears. Consequently, this suggests that the associated force contribution can be interpreted as a result of gust vorticity even for objects of volume. An added mass force arises due to body acceleration, occurring in unidirectional or oscillatory motion. Because the gust encounter involves no such body acceleration (apart from the initial start to achieve the free-stream velocity) it seems therefore more appropriate to attribute this force to gust vorticity instead of added mass. 
While this distinction may seem a somewhat pedantic question of semantics, it could become significant when attributing forces to vorticity external to the body, as any inclusion of gust shear layer vorticity as well as an added mass effect would lead to 'double-counting' the same physics. This also applies to other problems where vorticity is shed from more than one source, for example from two individual wings or when measurement data includes the boundary layer in a wind tunnel.

\section{Force Results}

The force balance measurements $C_{l}$ force balance for a surging cylinder encountering a sharp-edged gust at nominal gust ratios of $0.5,1$ and 1.5 are shown in figure 18 . For all gust ratios, $C_{l}$ rises quickly when the cylinder enters the gust. It increases to a maximum soon after the cylinder is completely immersed in the gust at roughly $x / D=1.5$, after which it decreases. Furthermore, a small positive force exists whilst the cylinder is upstream of the gust. This is likely caused by the transverse velocity upstream of the first shear layer, as seen in figure 12

Figure 18 also includes the non-circulatory corrected Küssner force $C_{l}^{n c}$ Küssner, assuming rigid infinitely thin shear layers as well as the non-circulatory smeared Küssner force, $C_{l_{\text {Küssner }}^{n c, ~ s m e a r e d ~}}$. As outlined in section II.B for the latter, the gust shear layer thickness is set to $0.5 \mathrm{D}$ and the transverse velocity upstream of the first gust shear layer is assumed to increase linearly from zero to a maximum of $0.15 G R$.

Furthermore, the experimentally determined non-circulatory gust force $C_{l g u s t}^{n c}$, accounting for the force contributions due to the rate of change of $\gamma_{g u s t}^{n c}$ and the shear layer deflection, equation 9 , is also given in figure 18 . This force is calculated from the measured flow field as described in section II.B.2 and is only computed for gust entry. During gust exit, significant shear layer and cylinder-shed vorticity leaves the field of view, which makes a force computation impossible. It should further be noted that $C_{l g u s t}^{n c}$ is subject to considerable uncertainty due to the need to manually distinguish between cylinder-shed and gust vorticity. Moreover, as vorticity sheds from either side of the cylinder this can entrain gust shear layer vorticity, further complicating the subsequent identification of both. The respective force history should therefore, not be taken as an exact quantitative result but rather be used as a qualitative indication of the development of the force contribution.

It was previously observed that the vortex sheet contribution from the gust shear layers $\gamma_{g u s t}^{n c}$ matches closely to the theoretically expected result once the cylinder is fully inside the gust. Therefore, it could be assumed that the experimental non-circulatory gust force contribution $C_{l_{g u s t}^{n c}}^{n c}$ should also match the smeared non-circulatory Küssner

force $C_{l}^{n c \text {, smearser }}$. The experimental and theoretical non-circulatory gust forces are compared to the measured force history in figure 18 , where it can be seen that: 


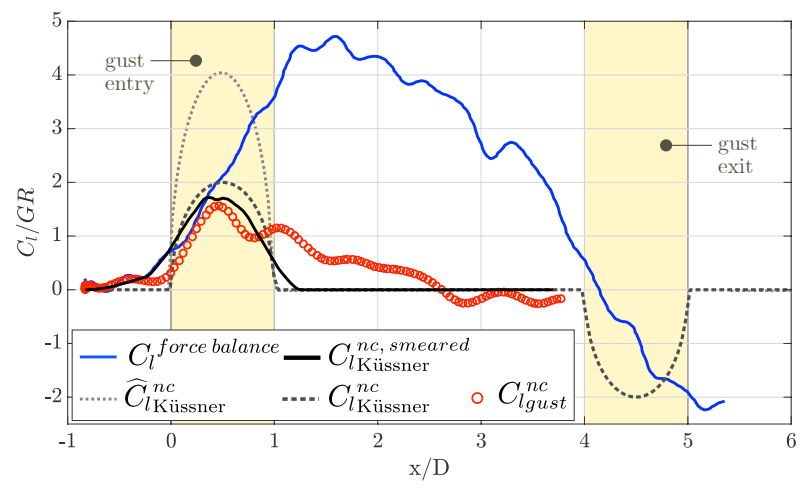

(a) Case 1, nominal $G R=0.5$.

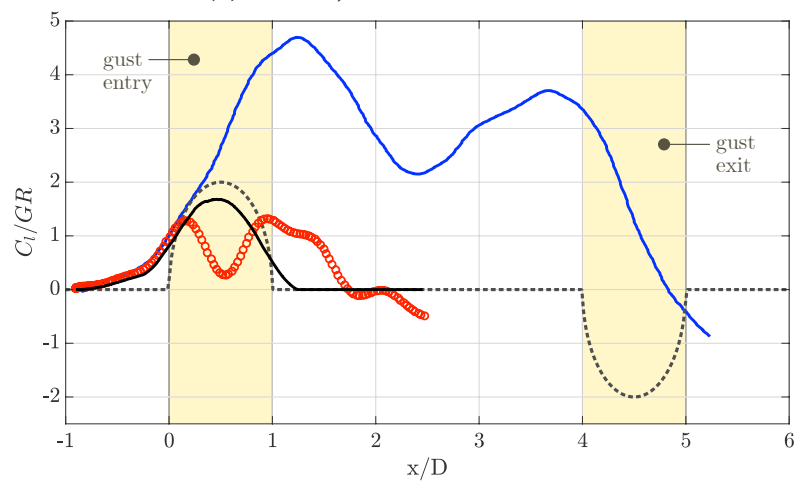

(c) Case 3, nominal $G R=1.5$.

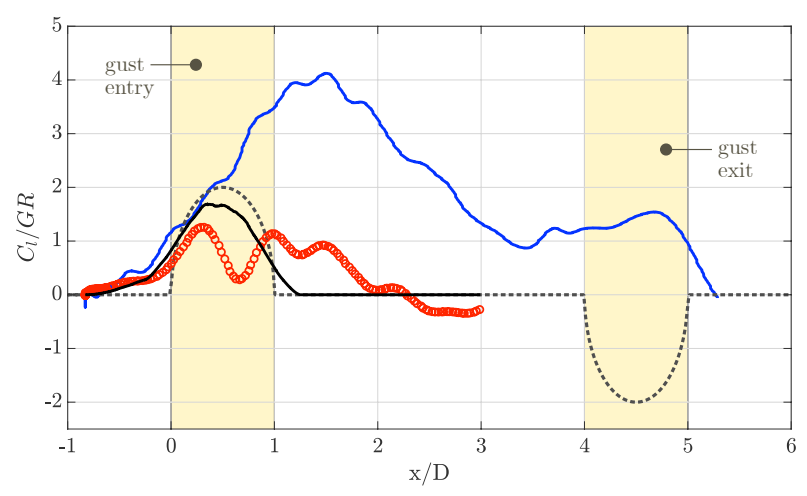

(b) Case 2, nominal $G R=1$.

Fig. 18 Theoretical and experimental forces acting on the cylinder.

- The corrected and smeared non-circulatory Küssner force, which include the volume correction described in section II.A (dark grey dashed and black line), are of the right order of magnitude during gust entry. The uncorrected non-circulatory Küssner force (light grey dotted line) included in figure $18 \mathrm{a}$ demonstrates that the respective force is far too great and thus implies that the correction, accounting for body volume as described in section II.A. is indeed necessary.

- The experimentally measured non-circulatory gust force (red circles) and the theoretical smeared non-circulatory Küssner force (black line) initially match the force balance measurements (blue line) for all gust ratios. This indicates that the non-circulatory gust force dominates the force response early on. Once flow separation occurs at approximately $x / D=0.37, x / D=0$ and $x / D=-0.1$ for $G R=0.5,1$, and 1.5 , respectively, the associated circulatory force contribution grows and begins to govern the overall force thereafter.

- Initially, as the cylinder enters the gust, the smeared non-circulatory Küssner force, $C_{l \text { Küssner }}^{n c \text { smeared }}$, is not too far off the experimental non-circulatory force, $C_{l g u s t}^{n c}$. This is the case especially when $G R=0.5$, where the force development matches until the cylinder is almost fully immersed in the gust. Only as the cylinder moves further into the gust, can a deviation between the three forces (red circles, black and blue line) be observed. These discrepancies appear to begin earlier and become more apparent for $G R=1$ and $G R=1.5$. Importantly, in all cases a measurable non-circulatory 
gust force, $C_{l g u s t}^{n c}$, remains even after the cylinder is fully inside the gust, whereas by this point the theoretical smeared Küssner force has vanished.

- A non-circulatory gust force is also present when the cylinder exits the gust. However, by now a considerable amount of shed vorticity is present in the flow field and the circulatory force contribution therefore dominates, obscuring any non-circulatory force effect.

The question now arises why the experimental non-circulatory force differs from the Küssner prediction for the two higher gust ratios and persists for longer in all cases. This is the case despite both theory and experiment arriving at the same non-circulatory gust vortex sheet once the cylinder is fully inside the gust. The answer appears to lie in the different growth rate of $\gamma_{g u s t}^{n c}$, since the force is in part linked to the rate of change of this vortex sheet. The distortion of the gust causes $\gamma_{\text {gust }}^{n c}$ to take longer before it reaches the theoretical distribution, hence widening and reducing the force peak.

Nevertheless, the force history of $C_{l g u s t}^{n c}$ in figure 18 implies that theoretical predictions assuming rigid shear layers can provide an approximate estimate of the non-circulatory Küssner force, especially at low gust ratios. This is shown by the match between the experimental force data, $C_{l g u s t}^{n c}$, and the theoretical prediction of the non-circulatory gust force, $C_{l_{\text {Küssner }}^{n c, ~ s m e a r e d ~}}$, when the cylinder has just entered the gust. However, with increasing gust ratio, the deflection of the gust

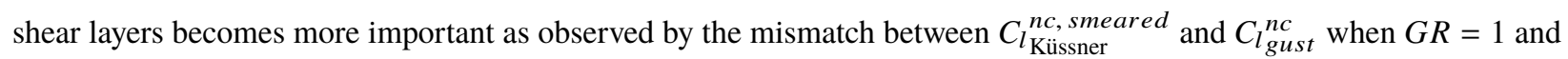
$G R=1.5$.

A further consequence of the increased gust ratio is that the circulatory force due to shed vorticity acts earlier and is more dominant. This is demonstrated by the earlier departure of $C_{l g u s t}^{n c}$, which excludes the effect due to any shed vorticity, from the force balance measurements for $G R=1.5$ compared to $G R=0.5$. A successful low order model predicting the forces acting on any body must therefore be able to predict both the non-circulatory component as well as that caused by flow separation.

\section{Conclusion}

For a flat plate entering a sharp-edged gust, an added mass term exists within the Küssner model. Von Kármán \& Sears [20] relate this force to the rate of change of a non-circulatory vortex sheet. Corkery \& Babinsky [15] showed for a thin flat plate that this vortex sheet forms because of the vorticity contained in the gust shear layers.

This paper investigates the non-circulatory force and the origin of the boundary layer vorticity for a body that has considerable volume. 
By applying potential flow concepts, a non-circulatory vortex sheet on the cylinder surface can be calculated, assuming two rigid gust shear layers. The existence of this vortex sheet is confirmed experimentally for varying gust ratios and kinematic surge combinations. This demonstrates that the concept of a non-circulatory vortex sheet arising from a transverse gust encounter can be extended from an infinitely thin flat plate to an arbitrary body of finite thickness.

The change in strength or distribution of the non-circulatory vortex sheet results in a force. In flat plate gust encounters it has been shown by others that this force is equivalent to the added mass force predicted by Küssner and von Kármán \& Sears. During gust entry this non-circulatory force matches well to the initial rise in $C_{l}$ experienced by a flat plate, however, for a cylinder this is not the case. In fact, it is a substantial over-estimation. This is because the force calculated from the rate of change of momentum caused by the growth of the non-circulatory gust vortex sheet does not account for the cylinder volume. It assumes that all of the transverse gust momentum in the region occupied by the cylinder is lost and is a result of the rigid shear layer assumption. Accounting for the change in momentum inside the cylinder leads to a closer match between the initial rise in force during gust entry and the non-circulatory gust force.

Comparing the theoretical prediction to measurements of the equivalent non-circulatory gust force indicates that shear layer thickness and deflection become more important with increasing gust ratio. At a gust ratio of 0.5 the non-circulatory gust force is initially well predicted by a modified Küssner force. However, for all cases, the experimental non-circulatory force persists for longer and circulatory gust forces dominate for most of the gust encounter. Even though the non-circulatory gust vortex sheet is ultimately identical to that predicted by theory when the cylinder is fully inside the gust, the growth rate is reduced as it takes significantly longer to develop. Consequently, this results in a lower and more spread out non-circulatory gust force development. Depending on the required accuracy of a low order model, shear layer thickness and distortion may therefore, need to be accounted for.

\section{Acknowledgments}

The authors would like to acknowledge the Engineering and Physical Science Research Council (EPSRC) for providing financial support, EP/M508007/1 and EP/N509620/1.

\section{References}

[1] Watkins, S., Milbank, J., Loxton, B. J., and Melbourne, W. H., “Atmospheric Winds and Their Implications for Microair Vehicles," AIAA Journal, Vol. 44, No. 11, 2006, pp. 2591-2600. https://doi.org/10.2514/1.22670. 
[2] Watkins, S., Thompson, M., Loxton, B., and Abdulrahim, M., "On Low Altitude Flight Through The Atmospheric Boundary Layer," International Journal of Micro Air Vehicles, Vol. 2, No. 2, 2010, pp. 55-68. https://doi.org/10.1260/1756-8293.2.2.55

[3] White, C., Lim, E. W., Watkins, S., Mohamed, A., and Thompson, M., "A Feasibility Study of Micro Air Vehicles Soaring Tall Buildings," Journal of Wind Engineering and Industrial Aerodynamics, Vol. 103, 2012, pp. 41-49. https: //doi.org/10.1016/j.jweia.2012.02.012

[4] Brunton, S. L., Rowley, C. W., and Williams, D. R., "Reduced-order unsteady aerodynamic models at low Reynolds numbers," Journal of Fluid Mechanics, Vol. 724, 2013, pp. 203-233. https://doi.org/10.1017/jfm.2013.163

[5] Stevens, P. R. R. J., and Babinsky, H., "Experiments to Investigate Lift Production Mechanisms on Pitching Flat Plates," Experiments in Fluids, Vol. 58, No. 1, 2017, pp. 1-17. https://doi.org/10.1007/s00348-016-2290-x.

[6] Eldredge, J. D., and Jones, A. R., “Leading-Edge Vortices: Mechanics and Modeling,” Annual Review of Fluid Mechanics, Vol. 51, No. 1, 2019, pp. 75-104. https://doi.org/10.1146/annurev-fluid-010518-040334

[7] Andreu-Angulo, I., Babinsky, H., Biler, H., Sedky, G., and Jones, A. R., "Effect of Transverse Gust Velocity Profiles," AIAA Journal, 2020, pp. 1-20. https://doi.org/doi.org/10.2514/1.J059665

[8] Hodara, J., Lind, A. H., Jones, A. R., and Smith, M. J., "Collaborative Investigation of the Aerodynamic Behavior of Airfoils in Reverse Flow," Journal of the American Helicopter Society, Vol. 61, No. 3, 2016, pp. 1-15. URL https: //doi.org/10.4050/JAHS.61.032001

[9] Sequeira, C. L., and Miller, R. J., “Unsteady Gust Response of Tidal Stream Turbines,” Oceans-St. John's IEEE, 2014, pp. 1-10. https://doi.org/10.1109/OCEANS.2014.7003026

[10] Saffman, P. G., Vortex Dynamics, Cambridge University Press, 1992.

[11] Eldredge, J. D., “A Reconciliation of Viscous and Inviscid Approaches to Computing Locomotion of Deforming Bodies," Experimental Mechanics, Vol. 50, No. 9, 2010, pp. 1349-1353. https://doi.org/10.1007/s11340-009-9275-0

[12] Graham, W. R., Pitt Ford, C. W., and Babinsky, H., “An Impulse-Based Approach to Estimating Forces in Unsteady Flow," Journal of Fluid Mechanics, Vol. 815, 2017, pp. 60-76. https://doi.org/10.1017/jfm.2017.45

[13] Corkery, S. J., Babinsky, H., and Graham, W. R., "Quantification of Added-Mass Effects Using Particle Image Velocimetry Data for a Translating and Rotating Flat Plate,’ Journal of Fluid Mechanics, Vol. 870, 2019, pp. 492-518. https://doi.org/10. $1017 /$ jfm.2019.231

[14] Gehlert, P., and Babinsky, H., "Linking the Unsteady Force Generation to Vorticity for a Translating and Rotating Cylinder," AIAA Scitech Proceedings, 2019. https://doi.org/10.2514/6.2019-0347.

[15] Corkery, S. J., and Babinsky, H., “An Investigation into the Added Mass Force for a Transverse Wing-Gust Encounter,” AIAA Scitech 2019 Forum, 2019. https://doi.org/10.2514/6.2019-1145 
[16] Wu, J. C., "Theory for Aerodynamic Force and Moment in Viscous Flows," AIAA Journal, Vol. 19, No. 4, 1981 , pp. $432-441$. https://doi.org/10.2514/3.50966

[17] Kelvin, L., “On Vortex Motion,” Trans. Roy. Soc. Edinb., Vol. 25, 1869, pp. 217-260.

[18] Küssner, H. G., "Stresses produced in Airplane Wings by Gusts," National Advisory Committee for Aeronautics, Vol. $654,1932$.

[19] Küssner, H. G., “Untersuchung der Bewegung einer Platte beim Eintritt in eine Strahlgrenze," Zentrale für techn.-wiss. Berichtswesen, Vol. 13, 1936, pp. 425-429.

[20] von Karman, T., and Sears, W. R., “Airfoil Theory for Non-Uniform Motion,” Journal of the Aeronautical Sciences, Vol. 5, 1938.

[21] Spalart, P. R., "Vortex Methods for Separated Flows,” Tech. rep., NASA, Moffett Field, 1988. https://doi.org/10.1007/3-54059280-6_96

[22] Chang, C. C., Yang, S. H., and Chu, C. C., "A Many-Body Force Decomposition with Applications to Flow about Bluff Bodies," Journal of Fluid Mechanics, Vol. 600, 2008, pp. 95-104. https://doi.org/10.1017/S0022112008000396

[23] Kang, L. L., Liu, L. Q., Su, W. D., and Wu, J. Z., "Minimum-Domain Impulse Theory for Unsteady Aerodynamic Force," Physics of Fluids, Vol. 30, 2018, p. 016107. https://doi.org/10.1063/1.5010008

[24] Westerweel, J., "Fundamentals of Digital Particle Image Velocimetry,” Meas. Sci. Technol, Vol. 8, 1997, pp. 1379-1392. URL http://iopscience.iop.org/article/10.1088/0957-0233/8/12/002/pdf

[25] Raffel, M., Willert, C. E., Wereley, S. T., and Kompenhans, J., Particle Image Velocimetry, $1^{\text {st }}$ ed., Springer, 1998. https://doi.org/10.1007/978-3-662-03637-2

[26] Wieneke, B., "PIV Uncertainty Quantification from Correlation Statistics," Measurement Science and Technology, Vol. 26, No. 7, 2015, p. 074002. https://doi.org/10.1088/0957-0233/26/7/074002

[27] Adrian, R. J., and Westerweel, J., Particle Image Velocimetry, Cambridge University Press, 2011.

[28] Corkery, S. J., Babinsky, H., and Harvey, J. K., "On the Development and Early Observations from a Towing Tank-Based Transverse Wing-Gust Encounter Test Rig,” Experiments in Fluids, Vol. 59, No. 9, 2018, pp. 1-16. https://doi.org/10.1007/ s00348-018-2586-0 